\title{
A Guide to Preprinting for Early Career Researchers
}

Authors: Cassandra L. Ettinger ${ }^{1^{*}}$, Madhumala K. Sadanandappa ${ }^{2}$, Kıvanç Görgülü ${ }^{3}$, Karen L. Coghlan $^{4}$, Kenneth K. Hallenbeck ${ }^{5}$, and Iratxe Puebla ${ }^{6}$

\author{
Affiliations: \\ ${ }^{1}$ Department of Microbiology and Plant Pathology, University of California, Riverside, Riverside, \\ CA, United States of America \\ ${ }^{2}$ Department of Molecular and Systems Biology, Geisel School of Medicine at Dartmouth, \\ Hanover, NH, United States of America \\ ${ }^{3}$ Comprehensive Cancer Center Munich, Klinikum rechts der Isar, Technical University of Munich, \\ Germany \\ ${ }^{4}$ George C. Gordon Library, Worcester Polytechnic Institute, Worcester, MA, United States of \\ America \\ ${ }^{5}$ TerraPrime, Danvers, MA, United States of America \\ ${ }^{6}$ ASAPbio, Cambridge, United Kingdom \\ *Address correspondence to Cassandra L. Ettinger (cassande@ucr.edu) \\ Competing interests declaration: \\ IP is an employee of ASAPbio, a non-profit organization promoting the productive use of preprints.
}

\section{Additional details}

cassande@ucr.edu

madhumala.k.sadanandappa@dartmouth.edu

kivanc.gorgulu@tum.de

kcoghlan@wpi.edu

k.hallenbeck@gmail.com

iratxe.puebla@asapbio.org

0000-0001-7334-403X

0000-0001-6071-365X

0000-0002-1613-1422

0000-0003-2784-6818

0000-0001-8662-2537

0000-0003-1258-0746

Keywords: preprints, early career researchers, how-to guide, open science, archiving, advice, life sciences 


\begin{abstract}
The use of preprints, research manuscripts shared publicly before the traditional peerreview process, is becoming more common in the life sciences. Early career researchers (ECRs) benefit from posting preprints as they are shareable, citable, and prove productivity. However, the decision to preprint a manuscript involves a discussion among all co-authors, and ECRs are often not the decision-makers. Therefore, ECRs may find themselves in situations where they are interested in posting a preprint but are unsure how to approach their co-authors or advisor about preprinting. Leveraging our own experiences as ECRs, and feedback from the research community, we have constructed a guide for ECRs who are considering preprinting - to enable them to take ownership over the process, and to raise awareness about preprinting options. We hope that this guide helps ECRs to initiate conversations about preprinting with co-authors and encourage them to preprint their future research.
\end{abstract}




\section{Introduction}

Preprints have attracted the attention of life scientists due to their growth in recent years and their role in facilitating the prompt sharing of research findings related to the COVID-19 pandemic (Fraser et al., 2021). Preprints support the rapid dissemination of research, accelerate scientific progress, and directly benefit individual researchers, particularly early career researchers (ECRs) - undergraduate students, graduate students, postdocs, research associates, research scientists, staff scientists, and other researchers. Preprints give ECRs more control over how and when to share their work than journal publications. In addition, preprints are a way for ECRs to present their research contributions to funding agencies and hiring committees while the work is still undergoing the editorial process at a journal.

While ECRs are often interested in open science and preprints, many find themselves in situations where the decision on how to publish their latest work does not lie solely with them. The decision on whether to preprint a manuscript involves a discussion among co-authors, and the ECR's advisor, the group leader, or the corresponding author will often make the final decision. Therefore, ECRs may find themselves in a situation where they would like to preprint but are unsure how to approach their advisor about preprinting. Drawing on our own experiences as ECRs and feedback from the research community, we have constructed the following guide for ECRs interested in preprinting their work. In this guide, we focus on: (1) what are preprints? (2) what are the preprinting trends in the life sciences? (3) how to approach conversations about preprints with co-authors? (4) what are common concerns about preprinting? (5) practical steps for depositing preprints, and (6) how to get involved with preprints more broadly. Besides raising awareness, we hope that the resources and suggestions in this article will be informative and helpful to ECRs to understand the advantages of preprints. 


\section{(1) Do your research - What is a preprint?}

A preprint is defined as a full draft version of a research manuscript shared publicly prior to the peer-review process (Mudrack 2021). Posting a preprint serves as a public, permanent disclosure of one's research. In patent terms it would serve as prior art, assigning a date in the scholarly record for any subsequent discussion of who found a particular result first. Preprints are assigned a persistent identifier, most commonly a Digital Object Identifier number (DOI), which allows them to become a permanent part of the scholarly record. The DOI records metadata for ease of discoverability. Many funders, such as the National Institute of Health $(\mathrm{NIH})$ in the US, the European Research Council, or the Australian Research Council, allow preprint citations in grant applications or reports (Kaiser 2017; Watson 2021). The preprint can be cited in subsequent papers furthering the scholarly record and making research results available in a timely manner.

Preprints are not new to the research community. In the 1960s, the NIH created the Information Exchange Groups (IEGs) to circulate copies of biological preprints. The IEGs ended up growing into seven different groups with a membership of over 3,600 participants and circulated 2,500 different documents, but by 1967 the IEGs were abandoned after several journal publishers refused to accept articles circulated as preprints (Cobb 2017). Physicists experimented with similar models, and in 1991, arXiv was founded as a repository for manuscripts in the physical sciences (ArXiv 2021). While physicists adopted preprints to disseminate work with colleagues, the use of preprints in the life sciences did not take off until the 2010s, with the start of bioRxiv in 2013 and initial signs of support by funders and publishers (Puebla et al., 2022).

An additional benefit of preprints is that they have no associated access barriers. Preprint servers are free-to-use and free-to-access, expanding the reach of readers for an article and enabling open science. Anyone can read, comment on, and cite preprints, thus facilitating early 
discovery and global public engagement (Maggio et al., 2018). Preprints also support an international and equitable scientific community - no paywall means that researchers can read and cite work they otherwise would not be able to access due to barriers caused by journal subscription fees.

\section{Preprint servers and landscape}

Preprints adoption in the life sciences started in the last decade with the launch of bioRxiv in November 2013. Since then, new preprint servers have appeared, such as AgriRxiv, $\underline{\text { Research }}$

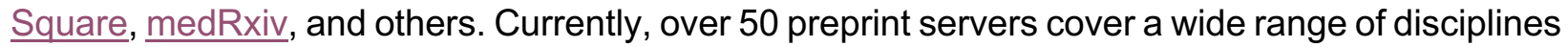
- for a list of preprint servers relevant to life sciences, biomedical, and clinical research, refer to the ASAPbio webpage (List of preprint servers). These servers follow different governance models and are operated by academic communities, academic institutions, or publishers. As Google Scholar and Europe PMC index many preprint servers such as arXiv, bioRxiv, Research Square, or medRxiv, keeping up to date with the latest preprints is relatively straightforward while searching for the literature on those platforms.

The number of cumulative submissions to various preprint servers in $2017-2021$ demonstrates increased acceptance of preprinting among life science researchers (Figure 1). bioRxiv, the largest biology preprint server, had cumulatively published 199,311 preprints by the end of 2021 (bioRxiv reports). 

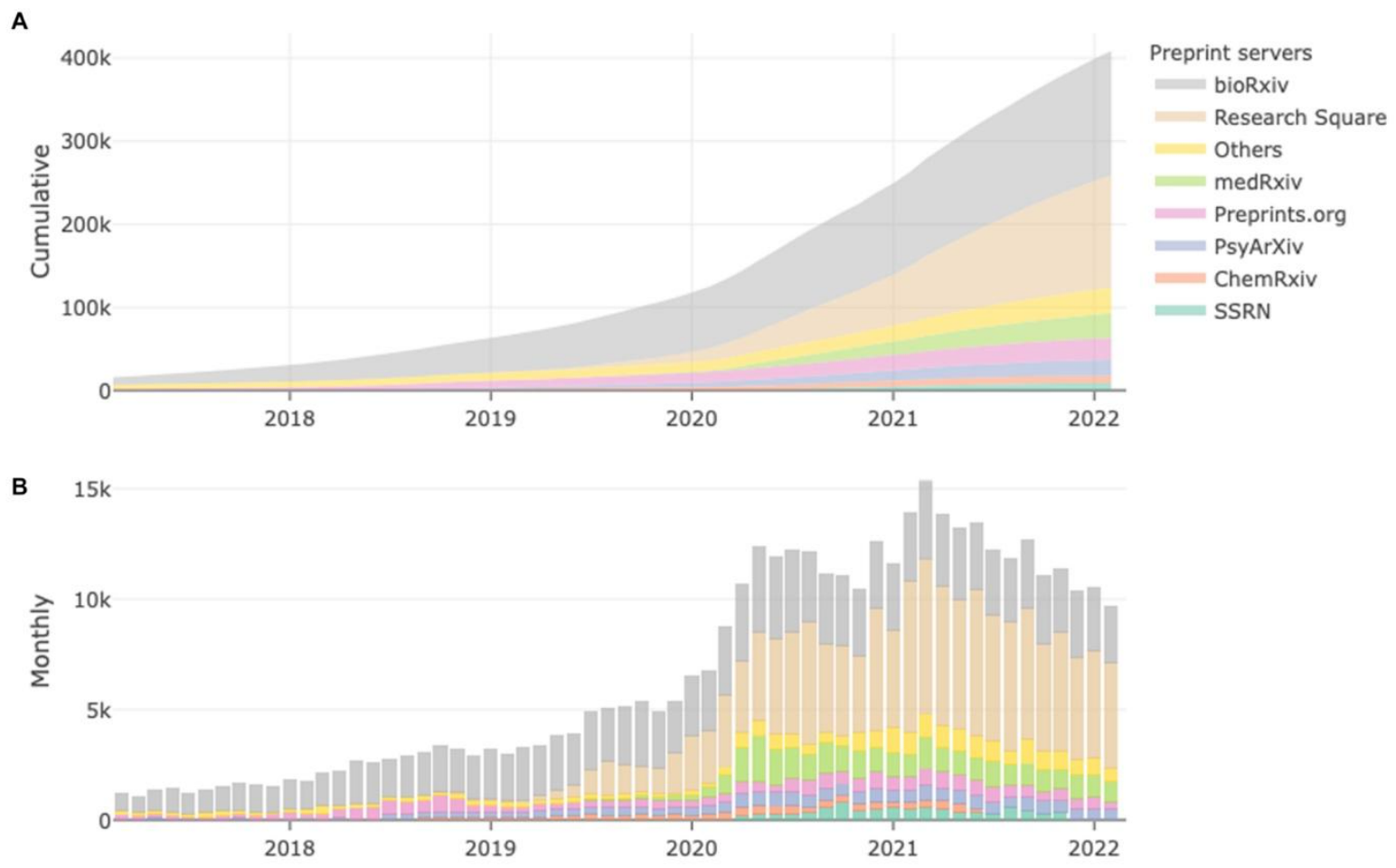

Figure 1: Preprint indexing in Europe PMC indicates an overall increase in preprints deposited between 2017 and 2021. (A) Number of cumulative and (B) monthly preprints hosted in different preprint servers. Figure reproduced from Europe PMC.

Using the bioRxiv Application Programming Interface, we retrieved preprint metadata from bioRxiv (January 2017 to December 2021) and medRxiv (June 2019 to December 2021), two of the largest life science preprint servers (Reported data was retrieved from the servers on March 2, 2022). We summarize how content has evolved in the last years and country-level representation at the servers. It is worth noting that trends might differ for other preprint servers not included here.

In January 2017, bioRxiv recorded 11,386 preprints since its launch, with 586 new submissions during that month alone. Remarkably, in the last five years, the number of monthly 
preprint deposits to bioRxiv increased to 2,965 in December 2021 (excluding revised manuscript submissions). Launched in June 2019, medRxiv started with 116 initial new preprint submissions, recording a cumulative total of 35,853 preprints by the end of 2021 (Figure 2A). Evidently, with the continued growth of preprints uploaded to these two servers, the preprint visibility in abstract views and manuscript downloads from bioRxiv and medRxiv also increased over time (Figure S1). Furthermore, there was a surge in preprinting COVID-19 related preprints during 2020, as well as broad scientific and public engagement with COVID-19 preprints during the pandemic (Fraser et al., 2021).

Based on the institutional affiliation of the corresponding author, we explored the countrylevel distribution for preprints in bioRxiv and medRxiv. As previously reported, we noticed disparities in adopting preprints across geographical regions (Abdill et al., 2020). The United States (39\%), followed by the United Kingdom (11.4\%), Germany $(6.02 \%)$, France $(4.37 \%)$, and China (4.16\%), occupy the top five places (Figure 2B). Researchers from other regions with solid levels of scholarly output - India (2.78\%), Japan (2.24\%), and Brazil (1.29\%) also represented content at the servers. Among African countries, South Africa $(0.25 \%)$, Nigeria $(0.12 \%)$, and Ethiopia $(0.11 \%)$ top the list in bioRxiv and medRxiv submissions. In June 2018, African researchers launched the pan-African open-access platform AfricArXiv, curating more than 800 submissions from 23 African countries. 

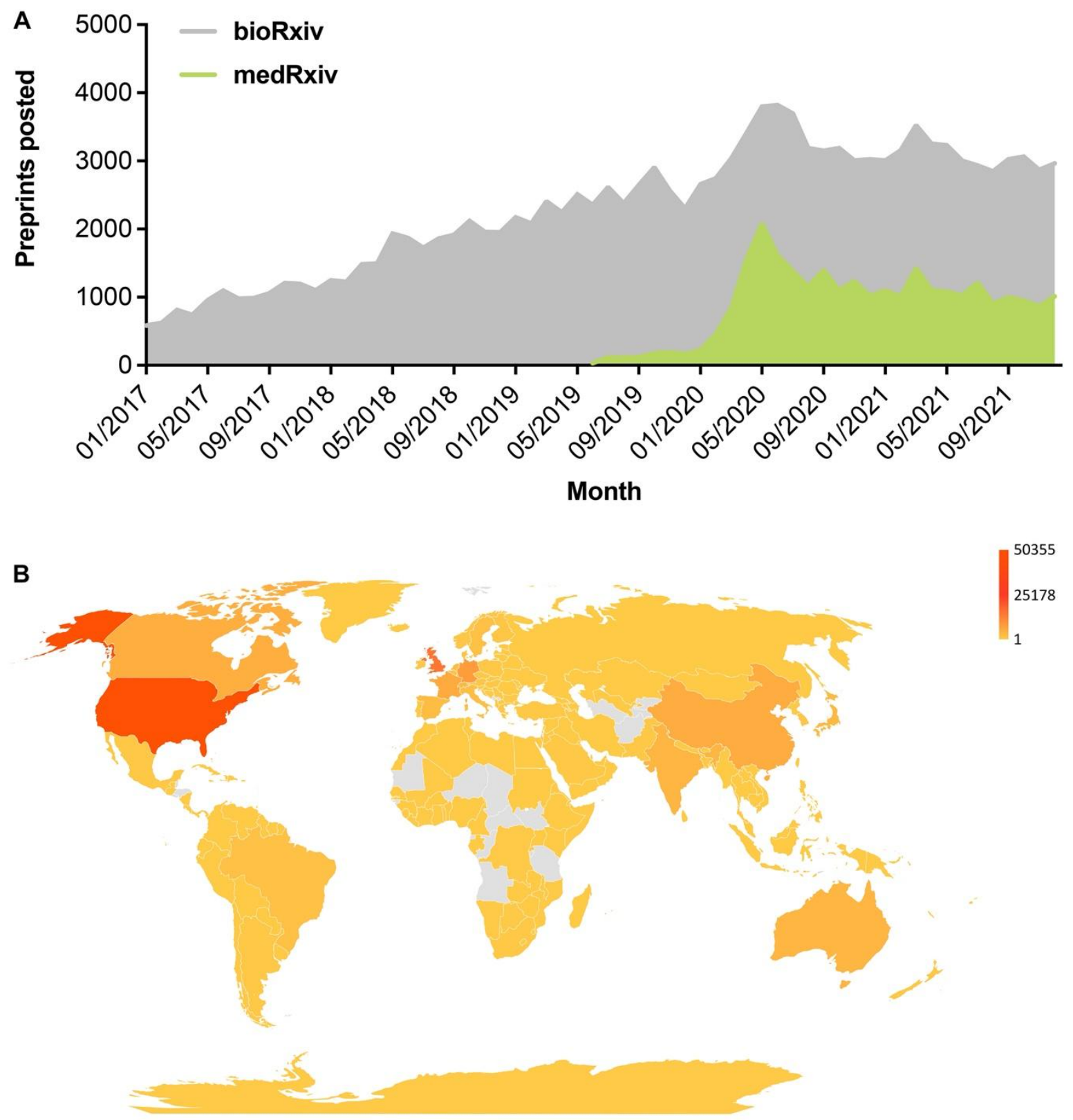

Figure 2: (A) Monthly submission of preprints to bioRxiv (grey - January 2017 to December 2021) and medRxiv (green - June 2019 to December 2021). (B) A heat map showing the country-wise distribution of preprints in both bioRxiv and medRxiv based on the institutional affiliation of the corresponding author. The color coding uses a log scale. (Data curated from bioRxiv and medRxiv- from servers launch till August 2021) 


\section{(2) Know your audience - How are different disciplines engaging with preprints?}

Consideration of preprint servers based on discipline, scope, policies, and readership is relevant to inform where to deposit your preprint, and in turn to maximize visibility for the work and opportunities for feedback from researchers in your specific field. Therefore, understanding the preprint trends in different disciplines can help identify how to best reach the audience for your work.

Within the life sciences, the adoption of preprints varies from one discipline to another. Looking at the data from bioRxiv and medRxiv, in 2020 and 2021, the most extensive collections in bioRxiv were Neuroscience, Microbiology, Bioinformatics, and Cell Biology, while in medRxiv the disciplines with strongest representation were Infectious diseases, Epidemiology, and Public and Global Health (Figure 3A; grey bars for 2020, orange bars for 2021). Preprints posted on bioRxiv and medRxiv go on to appear in a range of peer-reviewed journals, including PLOS ONE, eLife, Scientific Reports, Nature Communications, and BMJ Open (Figure S2). 

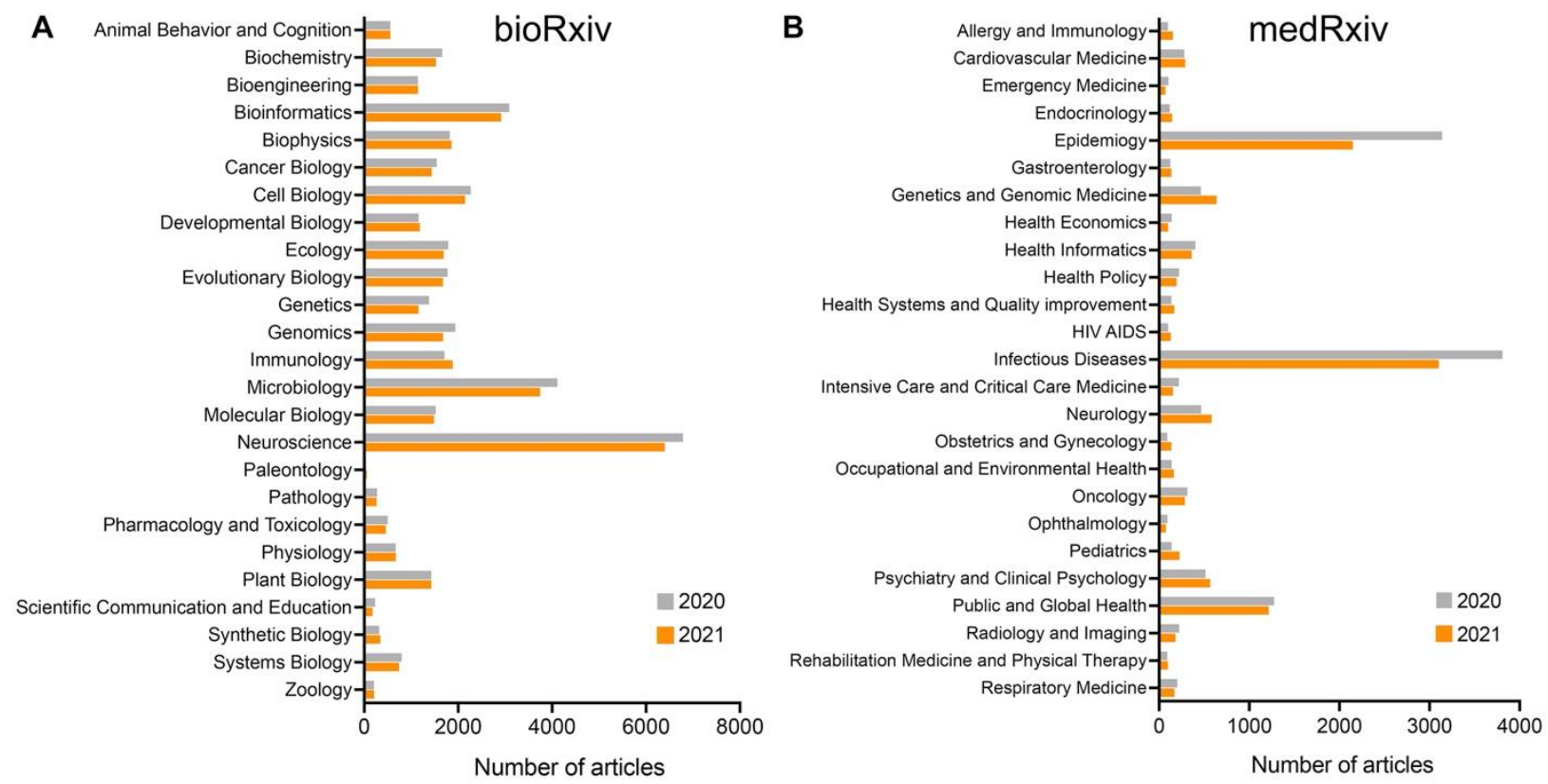

Figure 3: Discipline-level share of preprints deposited to (A) bioRxiv and (B) medRxiv. Data for 2020 and 2021 are shown in grey and orange, respectively. (Data curated from bioRxiv and medRxiv).

\section{Twitter survey on preprints}

To gauge the current impression around preprints within a portion of the scientific community, we conducted a Twitter poll (Figure 4, Table S1, responses to poll questions ranged from $n=19$ to $n=45)$. While the results of this poll are limited and not generalizable, they still provide valuable insights into current opinions on preprinting. Overall, $92.5 \%$ of respondents said they would recommend preprinting to ECRs. Many respondents were ECRs themselves when they published their first preprint $(77.7 \%)$. Of those respondents who had conversations about preprinting with colleagues who did not initially want to preprint, $42.1 \%$ could successfully convince their colleagues to preprint, while $57.9 \%$ could not preprint. For $20 \%$ of respondents, their manuscripts ended up in closed-access journals, meaning that preprinting here enabled them to have a green open-access option for the manuscript. Additionally, $61.8 \%$ of respondents 
reported that the data associated with their preprint was openly available online. Finally, $72 \%$ of respondents reported using a preprint in a grant or fellowship application. Although extremely limited and likely biased towards STEM ECRs as respondents, these Twitter surveys illustrate that ECRs are invested in the future of preprints and are thinking of ways to actively use preprints to benefit their research and careers.
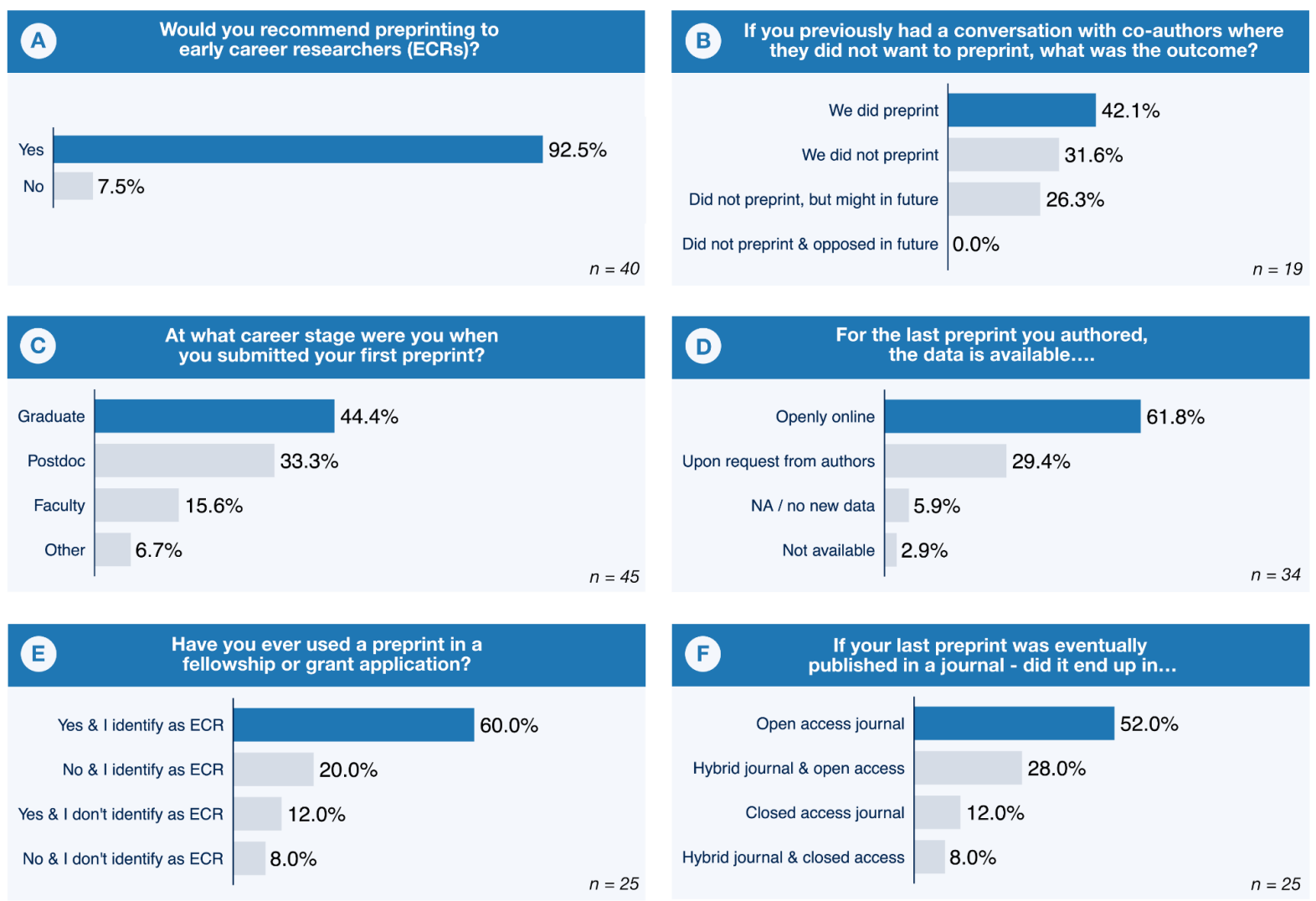

Figure 4: Twitter survey results. On August 30, 2021, we posted six multiple-choice survey questions (A-F) to Twitter to understand perceptions about preprints by some Twitter users and their experiences with preprinting (Table S1). Here we show the resulting percentage of responses to each question. The total number of respondents per question is notated in the bottom right corner of each panel, ranging from 19 to 45 responses per question. 
(3) I am thinking about preprinting my paper- how to approach it with my advisors and co-authors?

\section{Talking to your advisor, colleagues, and co-authors}

So, after considering all the above, you would like to preprint your paper; how to get started? As a first step, have a conversation with your advisor about preprinting your next paper. If you are unsure about where they stand regarding preprints, you can start by asking about their views on preprinting. If you have these discussions with your advisor or co-authors by email, we have provided some draft email structures to help you (Figure 5, Text S1). Here are a few things to consider:

- Keep it simple

- What are the requirements from your university or funders for communicating the work? Do they encourage preprints?

- What are your advisor's priorities for communicating the group's work?

- Provide examples of other researchers in your field who have preprinted

- Offer to provide additional resources or to seek further input about the use of preprints

If you are meeting with your advisor in person, even if you come prepared with all the answers, remember that your advisor may have questions that you did not anticipate or may still be unsure of what might be best for the work after your conversation. They may need time to mull over the options and get back to you. Not everything needs to be settled in one conversation; you can offer to gather more information on preprinting or their specific concerns to share with them and then continue the conversation at the next meeting. All authors must be on board to preprint the manuscript, so having these meetings early on can leave time for you to address concerns. 
In addition, make sure to consider the language and construction of the argument that you will use in your preprinting conversations. Try to use "I" language when discussing your goals and motivations and remind all parties how this aligns with your values or will benefit your career. If someone has a different opinion on preprinting than you do, investigate this opinion further by asking them how they reached that conclusion (e.g., did they have a colleague who was scooped?). Come prepared with resources to share and be aware of common concerns (see section 4 and Table 1), but do not pressure your advisor or colleagues to decide right away. Be ready to compromise and table the discussion to be followed up with in the future.

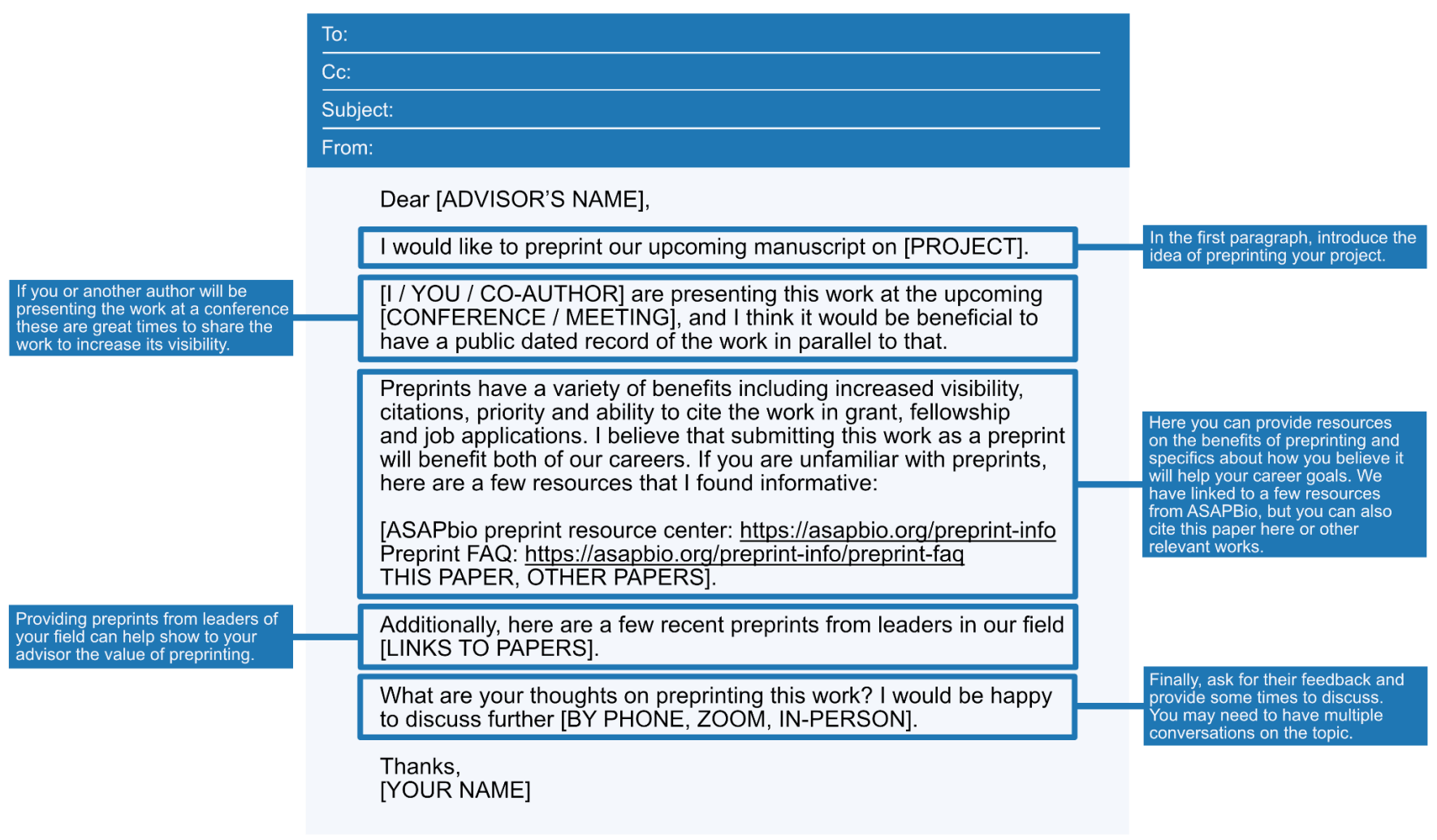

Figure 5: Draft email to one's advisor. An email template to help with initiating conversations about preprinting with one's advisor. We have included this same template and a template for emailing co-authors in text format in the supplementary materials (Text S1). 


\section{(4) Construct your argument - what concerns may come up in conversations about preprints?}

Several concerns or issues may come up in conversations with co-authors, colleagues, advisors, or others in the community. These issues might be influenced by research field, career stage, or experience. For example, those working in medical fields may raise concerns about sharing findings that may affect patients prior to peer-review; the stakes in patient treatment and public health are higher than in other fields. Preprint opinion may also differ depending on the level of acceptance of preprints in a discipline. For instance, in research fields with strong preprint adoption, it is unlikely to receive the response 'I did not see your work!' when you preprint. On the other hand, concerns about visibility or scooping may be more significant for fields with relatively lower adoption or acceptance of preprints.

We outline below (Table 1) some of the concerns or questions that may arise during discussions about preprints. In addition, we explore two of the most common themes in greater detail: scooping and sharing the work before the journal peer-review process.

Table 1: Examples of concerns or questions that may come up in conversation with your coauthors about preprints, along with information and considerations to raise in response when making a case for preprinting.

Concern / Question

'If we preprint the paper before publishing in a journal, we'll be scooped!'
Things to consider

Why preprint
Posting a preprint is not different from journal publication or presenting unpublished work at a conference.
A preprint provides a time-stamped permanent record of the work.

Journal scoop-protection policies recognize the date of preprint deposition. 
However, a preprint is citable and shareable.
The visibility afforded by a preprint can result in opportunities for a new collaboration.
'What if there are errors in the preprint?'
Prepare a preprint carefully before posting, just like you would for journal submission.

Compared to traditional peer-review, having more eyes and receiving feedback from the research community can help to strengthen the manuscript.

As preprint servers allow versioning, it is easy to deposit a revised preprint.

If we post a preprint, the journal will reject it as prior publication'
Preprint editorial policies vary across journals, before posting a preprint check the policy of the journal where you plan to submit the manuscript.
'If we post a preprint, attention will go to the preprint and will lessen the impact of the article once it appears in a journal'
The published journal article can be linked with the preprint record; some servers (bioRxiv, medRxiv) process this automatically, while in others direct requests can be made. Additionally, Google Scholar allows merging a preprint with its published version to calculate total citations across both.
Most journals in the life sciences now accept preprints. Many publishers operate or have partnerships with preprint servers.

When you preprint, you are giving the community a head start to build on your work - studies have shown that journal articles with an associated preprint receive more attention on social media and citations.
'Posting a preprint is one more thing to do when I am already busy preparing the journal submission'
Preprint servers allow flexibility in format, you can share your work when you are ready, without the hassle of formatting per journal requirements.
Some journals operate workflows that allow easy transfer from the preprint server to the journal, saving work in the submission process. Further, some journals include options to preprint during the initial journal submission.
'You need the journal publication for your career, why bother preprinting in addition?'

'If we preprint the paper, we will not be able to complete our patent application'
The preprint is a citable record that can be immediately included in your $\mathrm{CV}$.
While the manuscript is in the editorial process, a preprint provides proof of productivity, and several funding agencies accept preprints for grant applications.

Before preprinting discuss the possibility with the technology transfer office at your institution. disclosure, like journal publications. 


\section{Concern \#1: l'll get scooped}

A common concern among researchers is the risk of scooping - another competing group will see the preprint and rush to publish their results in a journal before the preprint authors have an opportunity to do so themselves. Interestingly, there is no evidence that the prevalence of scooping in preprints is higher than in the context of journal publications. For instance, in the 2019 bioRxiv survey, only $1.25 \%$ of the respondents indicated that posting a preprint negatively affected their priority claim for the work (Sever et al., 2019).

More remarkably, researchers have used their preprints as an opportunity to initiate collaborations with other groups in the field or to coordinate the publication of their work together, thereby avoiding concerns about priority claims. For example, Dr. Josh Hardy discussed how upon seeing a preprint from another group, they got in touch with the preprint authors. The two groups coordinated the journal publication of their respective papers, which ended up appearing in the same journal (Hardy, 2021).

Preprinting allows researchers much more control of when they disseminate their work and is thus an opportunity to prevent being scooped while waiting for the paper to be published in a journal. In addition, preprints provide an avenue for researchers in rapidly moving fields to promptly share their work with their community, where the delay associated with peer-review may come at the cost of priority. In the bioRxiv survey, $28 \%$ of respondents stated that preprints helped them stake a priority claim in their field (Sever et al., 2019).

\section{Preprints enhance visibility}

Visibility is an important element in the context of scooping concerns: preprints must be readily discoverable by researchers in the field, which in turn, allows attributing credit to the 
authors. Will the preprint be seen by colleagues in the field? Or is there a risk that the preprint may be overlooked, and competitors may not cite it?

In the bioRxiv survey, $74 \%$ of respondents stated that preprinting increased awareness of their research (Server et al., 2019). Preprints are readily searchable online, as indexing services and literature search tools increasingly incorporate them (Scopus, Google Scholar, Europe PMC, and Crossref all index preprints). In addition, authors can quickly disseminate preprints on social media platforms. For example, Twitter plays an important role in increasing the visibility of preprints, with many research groups sharing their latest preprints via Twitter or commenting on colleagues' latest preprinted work. Furthermore, social media platforms can allow scientists to immediately measure the community's reactions and engagement with the work, through the number of tweets, re-tweets, and likes the preprint receives. For example, the most tweeted preprints from bioRxiv and medRxiv in mid-September 2021 showed 520.5 tweets on average per day in the prior 30 days (Table S2).

In addition, studies have shown that posting preprints results in more attention on social media and a higher number of citations for the article once it appears in a journal. Altmetric scores are generally higher for articles deposited as preprints, with journal publications that have associated bioRxiv preprints receiving more mentions on blogs and Wikipedia than non-deposited articles, as well as more mentions in Twitter or Mendeley (Abdill et al., 2019a; Fraser et al., 2020); COVID-19 preprints have also been widely reported in the lay media (Fleerackers et al., 2021). The early accrual of citations for the journal publication suggests that the community had already taken note of the preprint, which gave them a chance to consider the work as part of their own research in the time between the preprint appearing and the journal publication.

\section{Preprints establish priority}


An important step in the research process is to disseminate your findings to the scientific community, and in turn, be able to claim credit for the work. Recognition for research productivity is essential to establishing a reputation in the field, acquiring grants, and career progress. A preprint provides a permanent time-stamped record for the research findings in a much shorter timeline than a journal publication. Thus, when time is critical (e.g., when completing your thesis or finishing a project before moving to another position), preprinting can greatly benefit ECRs.

In the coming years, life scientists might use preprints as a channel to establish priority, a shift like the physics community (Vale et al., 2016). In support of this idea, several publishers such as EMBO Press, PLOS, and eLife have 'scoop protection' policies that recognize the date of the preprint deposition.

Researchers often worry about the potential risk of scooping when they present their preliminary findings in conferences or symposiums. Attendees could use the information they heard at the conference and scoop the presenter, but as the information would have been available only to the conference attendees, it would be difficult to establish who did what when. Depositing a preprint before the conference presentation records the priority claim and protection from scooping.

\section{Preprints are citable}

A tangible benefit of preprints is that they are citable and can prove productivity for prospective funders. In our Twitter poll, $72 \%$ of respondents used a preprint in a grant or fellowship application (Figure 4). Many funding agencies now have policies that allow citing preprints as part of grant applications and reports (more information on funder policies at asapbio.org/funderpolicies). Following the example of the Australian Research Council that recently updated its policies to allow preprint citation in grant applications (Watson, 2021), we expect to see more 
funding agencies update their policies, recognizing the importance of preprints in the future. Besides funders, several research institutions have started to include preprints in their processes for hiring and promotion (see asapbio.org/university-policies).

\section{Concern \#2: My work hasn't been peer-reviewed yet}

Another common concern that may arise in conversations around preprints is sharing work before peer-review. Some researchers worry about disseminating their findings before going through the traditional peer-review process, which provides feedback on the work and can also address any errors before the broader circulation of the manuscript. Thus, the preprint should be carefully prepared before depositing it to the server, similar to journal manuscript preparation. To this end, ensure that all co-authors check the paper before posting and consider receiving feedback from colleagues prior to submitting the paper to the preprint server.

\section{Preprint feedback focuses on the science and not on journal fit}

An advantage of posting a preprint is that feedback received from the scientific community can help to improve the manuscript and is independent of subjective evaluations about journal fit. Incorporating community feedback into the manuscript can even increase the chances of eventual

publication. A preprint brings more eyes and a broader range of perspectives to the paper than the traditional two or three reviewers from the journal's peer-review - to that extent, it provides a robust mechanism to identify any issues before a paper enters the journal's process and valuable input on specific aspects of the manuscript (e.g., statistical analyses, a particular technique) or the interpretations of the data.

\section{Preprints enable journal-independent peer-review}

Several platforms offer feedback and evaluations on preprints. The peer-review process runs similarly to the traditional journal peer-review in some of these. For example, Review 
Commons, an initiative by EMBO Press and ASAPbio, allows researchers to submit their preprint for peer-review prior to journal submission. Review Commons has partnered with 17 affiliate journals - the Company of Biologists, EMBO Press journals, PLOS, eLife, Journal of Cell Biology and Molecular Biology of the Cell - that agreed to use the reviews provided by Review Commons to inform their evaluation and editorial decision, thus avoiding multiple review rounds. Review Commons requires the authors to post a preprint before the manuscript is submitted to an affiliate journal.

Services such as Review Commons and Peer Community In involve the review of preprints in a process coordinated by an editor or similar role. On the other hand, other platforms, such as PREreview and PubPeer, allow any community member to provide comments or reviews (Table 2). 
Table 2: Preprint commentary and review platforms and their characteristics. Information for the different platforms is based on the records available at Relmagine Review.

\begin{tabular}{|c|c|c|c|c|c|c|}
\hline Platform & $\begin{array}{l}\text { Review } \\
\text { requested } \\
\text { by? }\end{array}$ & $\begin{array}{l}\text { Reviewer } \\
\text { selected } \\
\text { by? }\end{array}$ & $\begin{array}{l}\text { Review } \\
\text { coverage }\end{array}$ & $\begin{array}{l}\text { Reviewer } \\
\text { identity } \\
\text { known }\end{array}$ & $\begin{array}{l}\text { Public } \\
\text { interaction }\end{array}$ & Recommendation \\
\hline Wlaudit & Non-authors & $\begin{array}{l}\text { Self } \\
\text { nominated }\end{array}$ & Complete paper & Public & Included & Binary decision \\
\hline$\equiv$ PREREVIEW & $\begin{array}{l}\text { Non-authors; } \\
\text { authors }\end{array}$ & $\begin{array}{l}\text { Self } \\
\text { nominated }\end{array}$ & $\begin{array}{l}\text { Complete paper } \\
\text { Specific aspects }\end{array}$ & $\begin{array}{l}\text { Public; no- } \\
\text { one }\end{array}$ & Included & Other scale or rating \\
\hline Th: PUBPEER & Non-authors & $\begin{array}{l}\text { Self } \\
\text { nominated }\end{array}$ & $\begin{array}{l}\text { Complete paper } \\
\text { Specific aspects }\end{array}$ & $\begin{array}{l}\text { Public; no- } \\
\text { one }\end{array}$ & Included & None \\
\hline ApreLights & Non-authors & $\begin{array}{l}\text { Self } \\
\text { nominated }\end{array}$ & Complete paper & Public & Included & None \\
\hline publons & Non-authors & $\begin{array}{l}\text { Self } \\
\text { nominated }\end{array}$ & Complete paper & Public & Not included & None \\
\hline Peerage of Science & Authors & $\begin{array}{l}\text { Self- } \\
\text { nominated, } \\
\text { Editor, service, } \\
\text { or community }\end{array}$ & Complete paper & $\begin{array}{l}\text { Editor or } \\
\text { service }\end{array}$ & Included & Binary decision \\
\hline $\begin{array}{l}\mathrm{PCI} \\
\text { Peer Community in }\end{array}$ & Authors & $\begin{array}{l}\text { Editor, service, } \\
\text { or community }\end{array}$ & Complete paper & $\begin{array}{l}\text { Editor or } \\
\text { service }\end{array}$ & Not included & Binary decision \\
\hline$\underset{\text { commons }}{\text { ReVlew }}$ & Authors & $\begin{array}{l}\text { Editor, service, } \\
\text { or community }\end{array}$ & Complete paper & $\begin{array}{l}\text { Editor or } \\
\text { service }\end{array}$ & Not included & None \\
\hline elife & Authors & $\begin{array}{l}\text { Editor, service, } \\
\text { or community }\end{array}$ & Complete paper & $\begin{array}{l}\text { Editor or } \\
\text { service }\end{array}$ & Not included & Binary decision \\
\hline
\end{tabular}

Public comments posted on the preprint can also help inform and positively shape the editor's decision upon manuscript submission to a journal. Some journals such as PLOS Genetics, Proceedings of the Royal Society B, and Open Biology have appointed preprint editors, who check the latest preprints to solicit submissions to their journals. 


\section{(5) Next steps - how to preprint your paper?}

Once you have your co-authors green light to preprint the work, here are a few actionable steps to complete the preprint deposition (Figure 6):

Preprint server. First, you need to choose a preprint server for your manuscript (Figure 6). Think carefully about your audience and what server will best reach the targeted audience (see section 2). If you plan to submit the manuscript to a journal, familiarize yourself with the journal's editorial policies about preprints. Some journals specify the preprint servers that they accept for preprint deposition.

\section{Preprint Submission Checklist}

$\square$ Choose a preprint server

$\square$ Double check journal requirements and preprint server policies

$\square$ Research preprint license options

$\square$ Prepare manuscript for publication according to journal and preprint server policies

$\square$ Discuss depositing any associated data to public repositories with your advisor and co-authors (optional)

$\square$ Decide on a preprint timing and submission plan with your advisor

$\square$ Submit your preprint

$\square$ Share on social media

Figure 6: Preprint submission checklist. A suggested checklist to help with preprint submission after having a successful conversation and the green light from advisors and co-authors to preprint. 
Preprint license: It is also important to think about the license you will apply to the preprint. You have several options - from no license (i.e., meaning you do not give default permission to reuse the work) to a range of Creative Commons licenses which designate the type of uses allowed for the work (asapbio.org/licensing-faq). While some preprint servers offer a range of license options (e.g., arXiv, bioRxiv, medRxiv, OSF Preprints), others require a CC-BY license (e.g., Research Square, preprints.org, SciELO Preprints) that allows reuse of the work with attribution.

Preprint preparation: In general, preprint servers are format agnostic, meaning they accept a single file of your manuscript in any format (for example, a single PDF file in the formatting style of the journal of your choice!) and then authorship information. In addition, data and other resources deposited in public repositories can be linked to your preprint. In fact, $61.8 \%$ of respondents on our Twitter survey reported that the data associated with their preprint was openly available (Figure 4). Some journals work with preprint servers, like bioRxiv, to allow for direct submission of your manuscript to a journal after posting to the preprint server. Similarly, during the manuscript submission, many journals will now ask if you would like the journal to send the manuscript to either a preprint server they partner with, like bioRxiv or Research Square, or an internal preprint server managed by the publisher, like Cell Sneak Peak, preprints.org or SSRN.

Preprint submission: Now that you've chosen a preprint server, license type, and prepared your manuscript, decide who will submit the manuscript and when it will be submitted. In the bioRxiv survey, authors preferred preprinting either before journal submission (42\%) or concurrent to journal submission (37\%) (Sever et al., 2019). After the preprint submission, don't forget to share your new preprint on social media (Heemstra, 2020; Cheplygina et al., 2020)!

\section{If your co-authors aren't interested in preprinting this time...}

Most respondents on our Twitter survey (57.9\%) unfortunately failed to convince their colleagues to preprint (Figure 4). Irrespective of the field, many researchers are still wary of 
preprinting, and it is understandable that other authors may have concerns or may need additional time to consider your request. Almost half of the respondents in our Twitter survey who were unable to convince their co-authors to preprint, indicated that their co-authors might be open to preprinting in the future. Offer to continue the conversation another time and suggest to them that it's worth keeping an eye on the latest preprints coming out in your field. You may also suggest you revisit the option of preprinting for another paper where they may view the stakes as less high. If your co-authors are still uninterested, there are still many other ways to get involved with preprints even if you are unable to preprint your current work.

\section{(6) What are other ways to get involved with preprints?}

Beyond providing an opportunity to promptly share your work and get credit for it, preprints also offer other benefits to your scientific career. For example, several communities with an interest in open science also support preprints and getting involved with one or more of those groups can be a way to expand your professional network and connect with other researchers in your discipline.

ASAPbio has an international community of researchers and others in the science communication space, who drive initiatives to support preprints and interact and support each other (more about the ASAPbio Community and how to join at asapbio.org/asapbio-community). ASAPbio also runs a Fellows program allowing participants to learn more about preprints and develop skills to drive discussions about the productive use of preprints in the life sciences. eLife coordinates an Ambassadors program, which aims to bring together ECRs interested in promoting change in academic culture and science communication.

If you are interested in developing your review skills, several options are also available. preLights is an initiative of the Company of Biologists to provide highlights on preprints. The highlights are contributed by ECRs who write summaries for preprints they find interesting. 
Preprint journal clubs are also an excellent opportunity to keep up to date with the latest research in your field and connect with others. If you are part of a local journal club, you can suggest incorporating preprints, if they are not already covered. If you do not have a local journal club, you can explore online options, e.g., PREreview coordinates live-streamed preprint journal clubs.

\section{Conclusions}

We hope that this guide will be a useful resource for readers, especially ECRs, interested in preprinting their work. In addition to exploring the current landscape of preprinting in the life sciences, we have discussed common concerns around preprints that might come up in conversations with colleagues. The tips provided in this article are useful for having conversations with advisors and co-authors about preprinting, including email templates and practical steps needed to preprint your work.

There are probably many tips and suggestions not covered in this piece. But as preprints continue to grow, so will our collective expertise, and the evidence around the use of preprints for science communication. We are excited to watch the preprinting community continue to grow and look forward to seeing more preprints and preprint engagement from ECRs in the coming years.

\section{Acknowledgements}

We thank ASAPbio for hosting the ASAPbio Fellows program and we are appreciative of the support received from the 2021 cohort of ASAPbio Fellows. We further thank bioRxiv and medRxiv for providing data on the country distribution of preprints at their servers. We are also grateful to Jessica Polka (ORCID: 0000-0001-6610-9293) and Samantha Hindle (ORCID: 00000002-3708-3546) for helpful suggestions on this manuscript. KG is supported by Deutsche Forschungsgemeinschaft (DFG) (Project no: 492436553). 


\section{References}

1. Abdill, R. J., Adamowicz, E. M., \& Blekhman, R. (2020a). International authorship and collaboration across bioRxiv preprints. ELife, 9, e58496. https://doi.org/10.7554/eLife.58496

2. Abdill, R. J., \& Blekhman, R. (2019a). Tracking the popularity and outcomes of all bioRxiv preprints. ELife, 8, e45133. https://doi.org/10.7554/eLife.45133

3. ArXiv. (2021) About arXiv | arXiv e-print repository..https://arxiv.org/about

4. BioRxiv Reporting. (2021). https://api.biorxiv.org/reporting/home

5. Cheplygina, Hermans, F., Albers, C., Bielczyk, N., \& Smeets, I. (2020). Ten simple rules for getting started on Twitter as a scientist. PLoS Computational Biology, 16(2), e1007513-e1007513. https://doi.org/10.1371/journal.pcbi.1007513

6. Cobb, M. (2017). The prehistory of biology preprints: A forgotten experiment from the 1960s. PLOS Biology, 15(11), e2003995. https://doi.org/10.1371/journal.pbio.2003995

7. Europe PMC. (2021). Preprints-About-Europe PMC. https://europepmc.org/Preprints\#questions-about-preprints

8. Fleerackers, Riedlinger, M., Moorhead, L., Ahmed, R., \& Alperin, J. P. (2021). Communicating scientific uncertainty in an age of COVID-19: An investigation into the use of preprints by Digital Media Outlets. Health Communication, 1-13. https://doi.org/10.1080/10410236.2020.1864892

9. Fraser, N., Brierley, L., Dey, G., Polka, J. K., Pálfy, M., Nanni, F., \& Coates, J. A. (2021). The evolving role of preprints in the dissemination of COVID-19 research and their impact on the science communication landscape. PLOS Biology, 19(4), e3000959. https://doi.org/10.1371/journal.pbio.3000959

10. Fraser, N., Momeni, F., Mayr, P., \& Peters, I. (2020). The relationship between bioRxiv preprints, citations and altmetrics. Quantitative Science Studies, 1(2), 618-638. https://doi.org/10.1162/qss a 00043

11. Hardy, J. (2021, July 8). Fear of being scooped is fuelling the replication crisis in research. Times Higher Education. https://www.timeshighereducation.com/opinion/fear-beingscooped-fuelling-replication-crisis-research

12. Heemstra, J. (2020). A Scientist's guide to social media. ACS Central Science, 6(1), 1-5. https://doi.org/10.1021/acscentsci.9b01273

13. International DOI Foundation. (2021). Digital object identifier system FAQs. https://www.doi.org/faq.html

14. Kaiser, J. (2017, March 24). NIH enables investigators to include draft preprints in grant proposals. Science Insider. https://www.science.org/content/article/nih-enablesinvestigators-include-draft-preprints-grant-proposals

15. Kirkham, J. J., Penfold, N., Murphy, F., Boutron, I., loannidis, J. P., Polka, J. K., \& Moher, D. (2020). A systematic examination of preprint platforms for use in the medical and biomedical sciences setting. BMJ Open 2020;10:e041849. https://doi.org/10.1136/bmjopen-2020-041849

16. Maggio, L.A., Artino Jr, A.R. \& Driessen, E.W. (2018). Preprints: Facilitating early discovery, access, and feedback. Perspect Med Educ 7, 287-289. https://doi.org/10.1007/s40037-018-0451-8

17. Mudrak, B. (2020, February). What are preprints, and how do they benefit authors? AJE Scholar. https://www.aje.com/arc/benefits-of-preprints-for-researchers/

18. Puebla, I., Polka, J., \& Rieger, O. Y. (2022). Preprints: Their evolving role in science communication. Against the Grain (Media), LLC. https://doi.org/10.3998/mpub.12412508

19. Sever, R., Roeder, T., Hindle, S., Sussman, L., Black, K.-J., Argentine, J., Manos, W., \& Inglis, J. R. (2019). bioRxiv: The preprint server for biology. 833400. https://doi.org/10.1101/833400 
20. Studenic, P., \& Ospelt, C. (2020a). Op0339 Altmetric-Does social media impact citation rate in rheumatology journals? Annals of the Rheumatic Diseases, 79(Suppl 1), 208-208. https://doi.org/10.1136/annrheumdis-2020-eular.5913

21. Vale, R. D., \& Hyman, A. A. (2016). Priority of discovery in the life sciences. ELife, 5, e16931. https://doi.org/10.7554/eLife.16931

22. Wang, X., Cui, Y., Li, Q., \& Guo, X. (2017). Social media attention increases article visits: An investigation on article-level referral data of peer journals. Frontiers in Research Metrics and Analytics, 2, 11. https://doi.org/10.3389/frma.2017.00011

23. Watson C. (2021). Australian funder backflips on controversial preprint ban. Nature News. https://doi.org/10.1038/d41586-021-02533-3 